\title{
The Design and Realization of Electronic-controlled Engine Fault Diagnosis Help System Based on Android
}

\author{
HAO BaoLan ${ }^{1, \text { a }}$, DOU lei ${ }^{2, b}$ \\ ${ }^{1}$ SHANDONG XIEHE UNIVERSITY \\ ${ }^{2}$ SHANDONG COLLEGE OF ELECTRONIC TECHNOLOGY \\ ${ }^{3}$ List all distinct addresses in the same way \\ a,b88407858@qq.com
}

Keywords: Android; engine; fault diagnosis; expert system

\begin{abstract}
For a long time, fault diagnosis expert system develops an important role in mechanical field, but the traditional expert system is generally operated on PC. However, it has poor mobile performance and can't meet the real-time and rapid outdoor requirements for fault diagnosis. With the rapid development of mobile internet technology in recent years, the mobile fault diagnosis expert system based on Android platform has no need to increase the extra hardware equipment. To gain real-time fault information and provide solutions has the important significance on studying the fault diagnosis expert system in the future engine field.
\end{abstract}

\section{Introduction}

The traditional fault diagnosis expert system for automobile engine accessories is developed and operated on PC, but it is short of the excellent mobility. With the popularity of the internet technology and smart mobile equipment, the studies on the movable fault diagnosis system are increasing, including engine fault diagnosis system based on Android and BP neural network, engine fault diagnosis system based on examples, and automobile fault diagnosis system based on mobile Bluetooth technology. In this paper, the author regarded Android smart phone as a platform tool and used the fault diagnosis of engine accessories as an example to access local servers and internet rapidly and conveniently.

\section{The Engine Fault Diagnosis Method Based on Neural Network}

The mathematical model of neurons is a classical and simplified model, shown in Figure 1. xj is the input of neurons, wji is the connection weight, $\theta \mathrm{j}$ stands for threshold value of ith neurons, and iy represents the output of ith neuron, shown in the Formula 1. Neurons firstly conducts linear weighted sum and then the transfer function $\mathrm{f}$ is conducted calculation and output results.

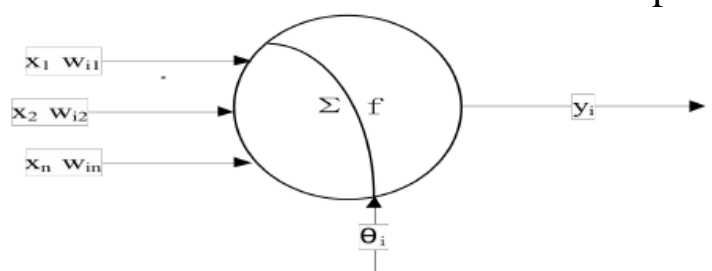

Fig.1 Mathematical Model of Neurons

$$
y_{i}=f\left(\sum_{j=1}^{n} W_{j i} x_{j}-\theta_{i}\right) \quad \text { Formula } 1
$$

B. The topological structure of BP network: The typical BP network structure is shown in Figure 2. The number of neurons in the input layer is $n$. The number of implicit neurons is $\mathrm{p}$, and the number of neurons in the output layer is $\mathrm{q}$. The activation functions stand for $\mathrm{fl}(\mathrm{x})$ and $\mathrm{f} 2(\mathrm{x})$. The network is defined as follows: 


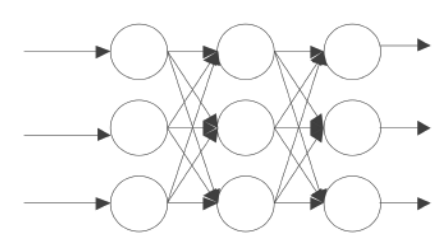

Fig.2 BP Network Structure

$\mathrm{xti}$ is the input of the neurons, $\mathrm{i}=1,2, \ldots \mathrm{n}$; wil is the weight from neurons in the input layer to the implicit neuron $\mathrm{l}, \mathrm{l}=1,2, \ldots, \mathrm{p}$; ul is the threshold of the implicit neuron; htl is the out of the implicit neuron; rlm is the connection weight from the implicit neuron to the neuron $\mathrm{m}$ in the output layer, $\mathrm{m}=1,2, \ldots, \mathrm{q} ; \mathrm{vm}$ is the threshold of the neurons in the output layer; $\mathrm{N}$ is the total number of samples; ytm is the output of the neurons in the output layer; dtm is the expected output; error function is formula 2.

$$
E_{t}=\frac{1}{2} \sum_{m=1}^{p}\left(y_{t m}-d_{t m}\right)^{2} \text { Formula } 2
$$

C. ELM topological structure of ELM neural network: There are $\mathrm{N}$ training samples, (xi, ti), $\mathrm{i}=1,2, \ldots \mathrm{N}$, where $\left.\mathrm{x}_{\mathrm{i}}=\left[\mathrm{x}_{\mathrm{i} 1}, \mathrm{x}_{\mathrm{i} 2}, \ldots, \mathrm{x}_{\mathrm{in}}\right]^{\mathrm{T}} \in \mathrm{R}^{\mathrm{n}}, \mathrm{t}_{\mathrm{i}}=\left[\mathrm{t}_{\mathrm{i} 1}, \mathrm{t}_{\mathrm{i} 2}, \ldots, \mathrm{t}_{\mathrm{in}}\right]^{\mathrm{T}} \in \mathrm{R}^{\mathrm{m}}\right)$, the number of neurons in the implicit layer is $\mathrm{k}$; the connection weight between the input nodes and ith implicit node is $\mathrm{w}_{\mathrm{i}}=\left[\mathrm{w}_{\mathrm{i} 1}, \mathrm{w}_{\mathrm{i} 2}, \ldots, \mathrm{w}_{\mathrm{in}}\right]^{\mathrm{T}}$, while the connection weight between ith implicit node and output nodes is $\mathrm{w}_{\mathrm{i}}=\left[\mathrm{w}_{\mathrm{i} 1}, \mathrm{w}_{\mathrm{i} 2}, \ldots, \mathrm{w}_{\mathrm{in}}\right]^{\mathrm{T}}$, bi is the threshold of the lth implicit node, shown in Figure 3 . The activation function is the mathematical model of $\mathrm{g}(\mathrm{x})$ standard single implicit layer's feedforward neural network is:

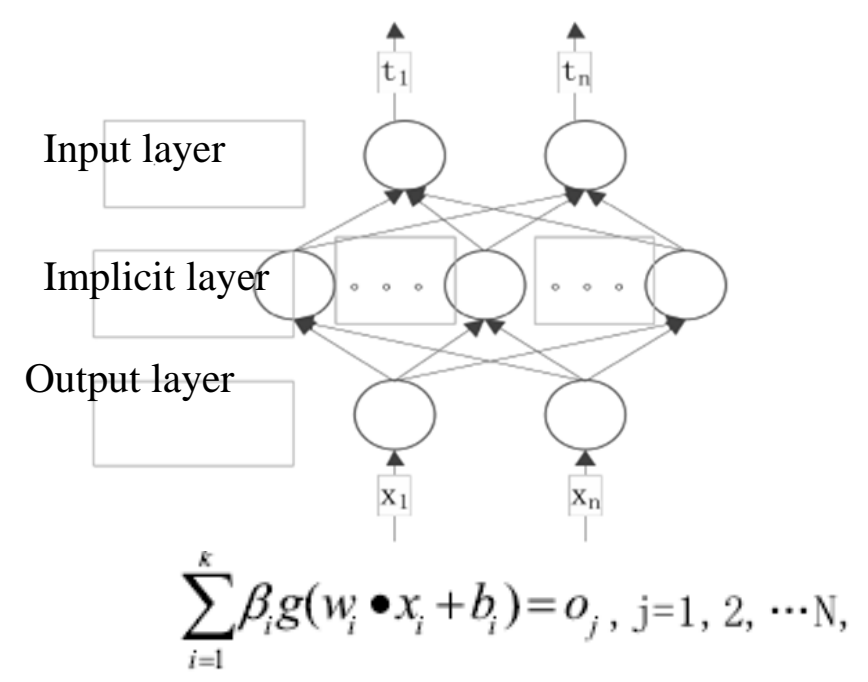

\section{Key Technology Analysis of Fault Diagnosis and Calibrated Software System}

A. Overall system design: The mobile automobile fault diagnosis and calibrated system based on Android is composed of two parts: lower computer and upper computer, shown in Figure 4. The lower computer is the Bluetooth controller module of CAN(for short Bluetooth-CAN module); through OBD-II interface and automobile ECU communication, the mobile automobile calibrated fault diagnosis and calibrated software system based on the Android is constructed.
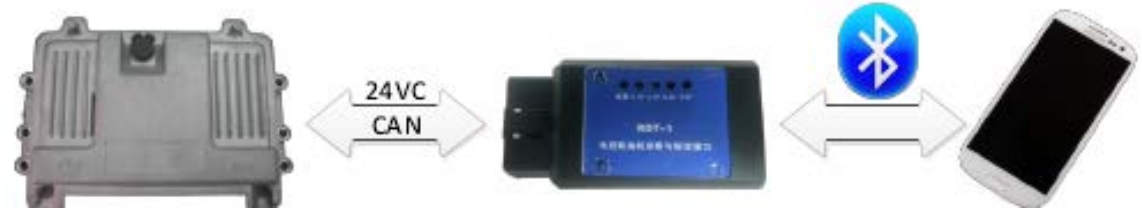

Fig.4 System Composition

(1) Workflow design of the system: the fundamental is the communication function of realizing upper computer mobile end and lower computer Bluetooth-CAN module. The server needs to embody vehicle status information and transfer it to Android smart phone. Android smart mobile 
phone client must be equipped with the active reading for vehicle traveling information; (2) System communication mechanism design: In the rules of the application layer or network layer, when message length exceeds 8 characters, SAEJ1939 provides the standard format for diagnosis definition. Shown in Table 1, the fault diagnosis code stipulated in the protocol has the suspected parameter number(SPN), fault mode symbol(FMI) and occurrence times(OC). Tab.1 SAE J1939 Protocol Diagnosis Fault Code Composition

\begin{tabular}{|l|l|l|}
\hline a & Suspected parameter number(SPN) & 19 bit \\
\hline b & Fault mode symbol(FMI) & 5 bit \\
\hline c & SAE reserved bit & 1 bit \\
\hline d & Occurrence times(OC) & 7 bit \\
\hline
\end{tabular}

B. System communication protocol design has four modules in this software system, including Bluetooth connection module, fault diagnosis module, dataflow module and calibration module. The fault diagnosis module is subdivided into the current fault diagnosis module and previous fault diagnosis module; calibration module is also divided into 8 modules in line with the ECU control module. The order format issued by the mobile client is shown in Figure 3(including code returning format). Each frame is stipulated as 12 bytes, including five parts(1) frame header: frame initiator of 2 bytes is used as the recognition area for the lower computer to accept data frame. The fixed identification OxAA55, 1 is alternated to reduce error code rate; (2) 0, frame length: 1 byte is used to stand for the character length of the data frame for whole frame message; (3) Data frame TAG ID: 4 bytes; in the order format, CAN2.012 bytes, so data frame in OxOco protocol belongs to the TAG ID in arbitration. In the code return format, OxAAFFFFFF is used for replacement; (4)PGN parameter number: 3bytes; in the order format, it is a part in the data frame field in CAN2.0 protocol. It is a 24-bit value, including reserved bit, data page, PDU format field and extended field. In code return format, OxFFFF55 is used for replacement. (5) frame tail: 2-byte end area is used as the identification area for the lower computer to accept the end of the data frame. The fixed identification Ox33CC is used alternation of Ox33CC to reduce error code rate.

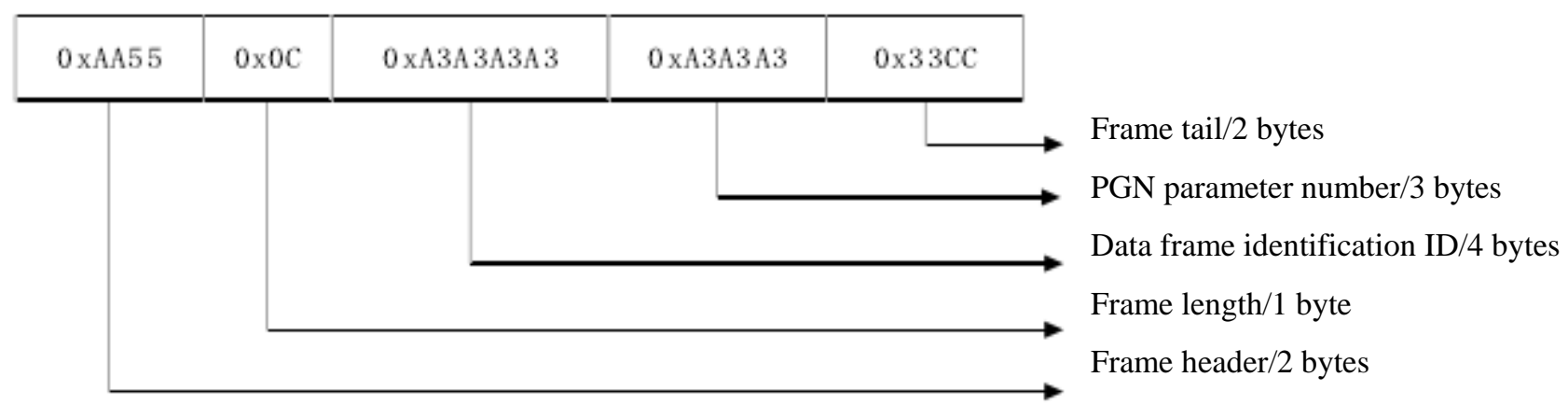

Fig.3 Customized Protocol Format of the Mobile Client

The data format received by the mobile client is shown in Figure 4. Each frame is stipulated as 16bytes, including 6 parts: (1) Frame header: 2-byte frame initiator is the identification area for the lower computer to accept the data frame; fixed identification Ox5A; 1 is used to alternate and reduce error code rate; (2) Frame length: 1 byte stands for the character length of data frame; whole frame message 16 bytes, thus Ox10o; (3) Data frame identification ID: 4 bytes; it is the TGA ID o of CAN data frame in the arbitration field; (4) PGN parameter code: 3 bytes; it is a part of CAN data frame in the data field; 24-bit value, including reserved bit, data page, PDU format field and group extended field. (5) data code: 4 bytes; the fault code in the fault module; the data flow code in the data flow module; the calibrated data in the calibrated module; (6) Frame tail: 2-byte end area is the identification area for the lower computer to accept the end of data frame; fixed identification OxCC33; 00, 11 is alternated to reduce error code rate. 


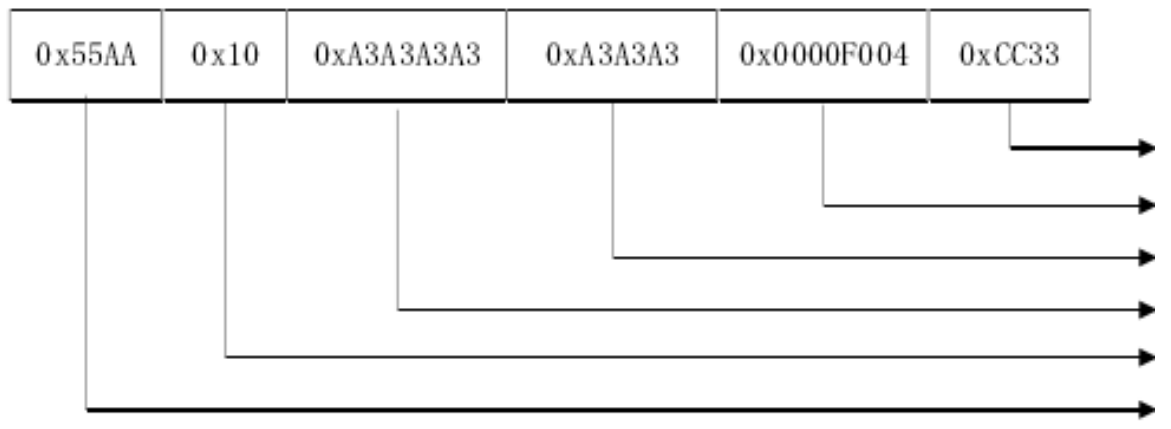

Frame tail/2 bytes

Data code/4 bytes

PGN parameter number/3

bytes

Data frame identification

ID/4 bytes

Fig.4 Bluetooth-CAN Customized Protocol Format

The message received by mobile client needs three processes of judgment auditing. After meeting conditions, it can be used as the effective message to display results in mobile client. If the auditing is not passed, mobile client will send order again. The lower computer should send it again.

\section{Fault Diagnosis and Calibrated System Software Design}

Android platform framework is similar to the other mainstream smart system. All of them apply the hierarchal framework, including Application, Android Runtime, Libraries, Application Framework and Linux Kernrl, shown in Figure 5.

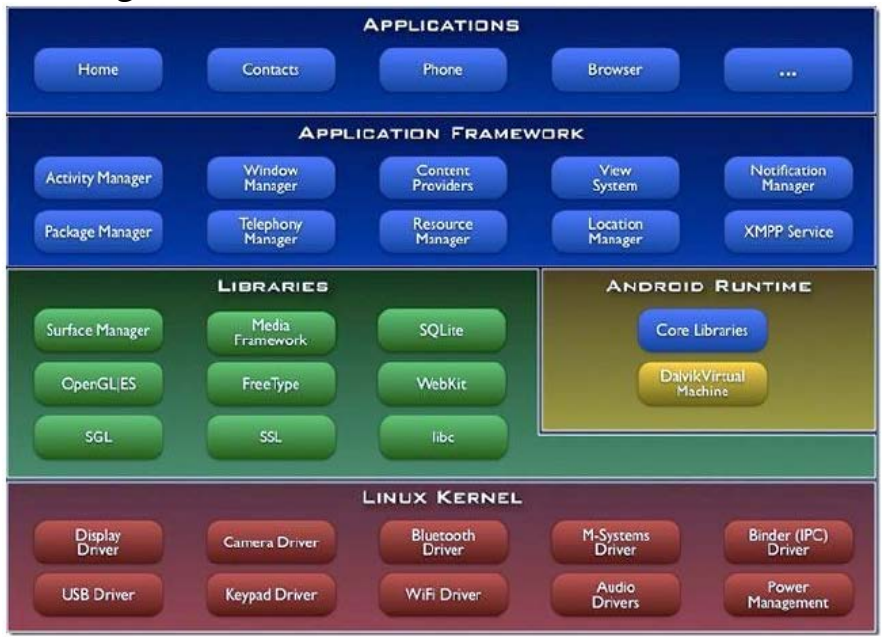

Fig.5 Android Operating System Framework

$<$ ?xml version=" 1.0" encoding="UTF-8"? $>$

The modular design pattenr must conform to each enterprise requirement, which will be neglected. In the interface design, UI design is the most important. Each type of module has the respective stipulated pattern design. Through constant perfection and constant comparison, Java code is used for realization in $\mathrm{xml}$ document. The pattern procedure of list control ListView is shown as follows:

<layer-list xmlns:android="http://schemas.android.com/apk/res/android" > $\quad<$ shape $>$ <solid android:color="\#D3D3D3" /><corners android:radius="12dp"/>

$<$ shape $>$ <item $>\quad<$ item android:bottom="3dp" androidaeft="3dp" android: top="3 dp" android:right="3dp"/> <shape $>$

$<$ solid android:color="\#FFEFD5"/> <padding androidaeft="4dp" androidaop="0.5dp"android:right="4dp" android:bottom="0.5dp"/> <corners android:radius=" 12dp"/> </shape $></$ item $><$ /layer-fist $>$

\section{Conclusions}

In the mobile internet era, fault diagnosis expert system has the new development direction. Differing from the fault diagnosis expert system based on Web technology, fault diagnosis expert system based on Android mobile platform can obtain several times of artificial solution efficiency as solving faults through the experimental capacity. The fault diagnosis expert system design scheme for engine 
accessories based on Android has the excellent effects. It is suitable for being promoted to the fault diagnosis fault system of the other industries.

\section{Acknowledgements}

This paper is supported by A Project of Shandong Province Higher Educational Science and Technology Program.No.J16LB70

\section{References}

[1] Meng Xianglei, the Application of Waveform Analysis in Engine Fault Diagnosis[J], Electronic Technology and Software Engineering, 2017, 01: 258;

[2] Wang Dongliang and Min Yongjun, the Correlation Feature Analysis and Diagnosis Optimization in the Electronic-controlled Engine Fault Dataflow[J], Agricultural Equipment and Vehicle Engineering, 2013, 12:56-60;

[3] Zhang Jiapei and Xu Ping, the Application of Short-time and Long-time Fuel Oil Correction in Unstable Idle Speed Fault Diagnosis Teaching of Electronic-controlled Engine[J], Industrial and Information Education, 2014, 04: 47-49;

[4] Wu Tao, Fan Yuqi, Zhou Chen and Chen Jinwei, the Study on the Measured Network System of Electronic-controlled Engine[J], Electronic Mass, 2015, 07:9-12+ 26;

[5] Wu Gang and Yuan Qingke, the Study and Prospect of Electronic-engine Engine System Fault Diagnosis Technology[J], Mechanical Manufacturing and Automation, 2017, 05: 243-245. 